ESJ Social Sciences

\title{
Développements et transformations digitales de l'enseignement supérieur au Liban : défis et perspectives
}

\author{
Sonia Messai-Farkh \\ Maha Badr \\ Fady Calargé
}

Université Libanaise, Administration Centrale-Badaro, Museum-Beyrouth

Doi:10.19044/esj.2022.v18n6p58

Submitted: 19 January 2022

Accepted: 22 February 2022

Published: 28 February 2022
Copyright 2022 Author(s)

Under Creative Commons BY-NC-ND

4.0 OPEN ACCESS

Cite As:

Messai-Farkh S., Badr M. \& Calargé F. (2022). Développements et transformations digitales de l'enseignement supérieur au Liban : défis et perspectives. European Scientific Journal, ESJ, 18 (6), 58. https://doi.org/10.19044/esj.2022.v18n6p58

\section{Resume}

L'objectif de notre recherche se veut une réflexion critique sur le rôle des enseignants de français pour publics spécifiques dans un contexte universitaire libanais post COVID-19. Elle s'inscrit donc dans le prolongement et l'approfondissement des réflexions sur l'enseignement/apprentissage en distanciel suite au confinement imposé depuis mars 2020. Pour mener cette étude, nous avons choisi de nous référer à un outil de modélisation appelé « Arbre de décision de réponse » issu de la théorie des «gestions de crise ». Nous l'avons appliqué au contexte de l'enseignement à distance dispensé au Liban suite à la crise sanitaire afin d'apporter des propositions opérationnelles et immédiates aux enseignants. De plus, nous avons mené conjointement des enquêtes par questionnaires, des entretiens semi-directifs et collecté des témoignages auprès des formateurs du supérieur au Liban. L'analyse jumelée de nos investigations a permis d'établir un état des lieux précis de l'intégration des TIC dans le système universitaire et de faire émerger des besoins prioritaires de formations. En effet, le corps professionnel exige une professionnalisation du métier d'enseignant pour faire face aux nouveaux défis liés à l'usage des TIC. Forts de ce constat, nous proposons un travail novateur et inédit : une proposition d'exploitation pédagogique en lien avec les arts visuels afin d'accompagner les enseignants dans l'enseignement en ligne. 
Mots-clés: TIC, Gestion de crise, enseignement, méthodologie, compétences langagières, COVID-19, art

\title{
Developments and Digital Transformations of the Lebanese Higher Education: Challenges and Perspectives
}

\author{
Sonia Messai-Farkh \\ Maha Badr \\ Fady Calargé \\ Université Libanaise, Administration Centrale-Badaro, Museum-Beyrouth
}

\begin{abstract}
The objective of our research is a critical reflection on the role of French teachers for specific audiences following the COVID-19 crisis in the Lebanese universities context. It is therefore part of the extension and deepening of reflections on distance teaching/learning following the lockdown enforced since March 2020. To conduct this study, we chose to refer to a modeling tool called "Decision tree for decision making" from the "crisis management" theory. We have applied it to the context of distance education provided in Lebanon following the health crisis in order to provide operational and immediate proposals to teachers. In addition, we jointly conducted questionnaire surveys, semi-structured interviews and collected testimonies from higher education trainers in Lebanon. The combined analysis of our investigations has made it possible to establish a precise inventory of the integration of ICTs in the university system and to identify priority training needs. Indeed, instructors require a professionalization of the teaching craftsmanship to face the new challenges related to the use of ICT. Based on this observation, we propose an innovative and original work: a proposal for educational exploitation in connection with the visual arts in order to support teachers in online teaching.
\end{abstract}

Keywords: ICT, Crisis management, teaching, methodology, language skills, COVID-19, art

\section{Introduction}

L'analyse des rapports qu'entretiennent les enseignants et les étudiants avec l'usage des TIC ouvre des perspectives heuristiques sur le plan de la recherche et de la formation en éducation. Ce sujet d'une importance primordiale est d'actualité ; cependant, il demeure peu exploré notamment dans les pays en voie de développement. Par conséquent, notre intérêt se porte sur l'utilisation par les acteurs pédagogiques des outils numériques et des 
canaux digitaux dans un contexte libanais. Notre hypothèse est la suivante : même si la situation l'oblige, la décision d'adopter l'enseignement en ligne semble nécessaire mais elle est contradictoirement hâtive dans la mesure où de nombreux facteurs empêchent, à la fois, administratifs, enseignants et étudiants, de s'adapter à un enseignement rarement utilisé au sein des universités libanaises. En effet, comme le précise Carey (2016), « implementing quality online education is easier said than done ${ }^{1}$ »

À partir de l'analyse jumelée d'enquêtes quantitatives et qualitatives, nous dresserons un état des lieux précis des utilisations des technologies numériques au service de l'enseignement/apprentissage. Dans notre étude, nous présenterons, dans un premier temps, la situation sanitaire aux niveaux mondial et local sur fond de la théorie de la « gestion de crises ». Ensuite, nous focaliserons notre recherche sur le contexte des universités libanaises pour retracer la genèse du déploiement de l'enseignement en ligne. Nous présenterons également les ressources et préparatifs mis en place par les universités afin d'assurer ce type d'enseignement. Pour ce faire, nous dégagerons plus particulièrement les représentations des enseignants quant :

- au processus d'intégration des TIC dans l'enseignement/apprentissage ;

- aux besoins de formations des ressources humaines.

Nous avons pris le parti de proposer une entrée aux cours de langue par le biais des arts visuels. En effet, les arts visuels, comme nous le verrons ultérieurement, ont joué un rôle majeur en temps de COVID-19 et ils constituent des ressources pédagogiques, culturelles et scientifiques pour enseigner et apprendre la langue française dans le supérieur.

Les analyses sont centrées sur l'apprentissage en ligne en situation d'urgence ; s'agit-il d'une transposition de l'enseignement sur la toile et d'une simple conversion en cours en ligne d'une série d'interactions cognitives ? Faudrait-il, impérativement, mettre en place de nouvelles pratiques enseignantes et par la suite concevoir les activités pédagogiques d'une manière spécifique ou plutôt encadrer les enseignants pour leur assurer une adaptation optimale à l'enseignement en ligne ? Quel(s) rôle(s) pourrait jouer l'art dans le processus de l'enseignement virtuel de la langue française ? Les réponses à toutes ces interrogations nous mènent à proposer des perspectives actionnelles adaptées au terrain pouvant suivre la perpétuelle évolution et la mutation numérique.

\footnotetext{
${ }^{1}$ Mettre en œuvre un enseignement en ligne de qualité est plus facile à dire qu'à faire (NT). Archyde, (2020, 21 mars). From Hong Kong to Barcelona, universities face the challenge of online education.

https://www.archyde.com/from-hong-kong-to-barcelona-

$\% \mathrm{E} 2 \% 80 \% 8 \mathrm{~B} \% \mathrm{E} 2 \% 80 \% 8 \mathrm{Buniversities-face-the-challenge-of-online-education/,}$
} 


\section{La COVID-19 : la genèse d'une crise aux multiples facettes}

La pandémie de COVID-19 a provoqué la plus grande perturbation des systèmes éducatifs du siècle présent. À la mi-avril 2020, 94\% des élèves et étudiants dans le monde ont subi l'impact de la pandémie, " soit 1,58 milliard d'enfants et de jeunes scolarisés du pré-primaire au tertiaire dans 200 pays » (Nations Unies, 2020). Au Liban, dans l'enseignement supérieur, les cours préenregistrés et les plateformes en ligne ont souvent remplacé l'enseignement en présentiel, mais certaines universités ont suspendu leurs cours en raison des faibles capacités et le peu d'infrastructures dont elles disposaient. La plupart des enseignants n'étaient pas préparés à assurer la continuité pédagogique et à s'adapter aux nouvelles formes d'enseignement (El Hage, 2020). D'ailleurs, les Nations Unies précisent dans une note de synthèse publiée en août 2020 que bien que la crise sanitaire soit une source d' " innovation dans le secteur éducatif » (Nations Unies, 2020) et qu'elle ait donné naissance à «des initiatives innovantes, qui ont permis la poursuite d'activités d'enseignement et de formation - à la radio, à la télévision ou sous la forme de kits pédagogiques pour la maison », elle "a révélé [toutefois] que la formation initiale et continue des enseignants devait être repensée de façon à mieux les préparer aux nouvelles formes d'enseignement » (Nations Unies, 2020).

À l'instar de beaucoup de pays, le Liban semble suivre le cycle des gestions de crises dans son secteur éducatif ; l'enseignement est amené à suivre le principe méthodologique de triangulation cyclique des données : de la préparation, à la lutte et au redressement. Si la riposte se traduit en premier par les protocoles de dépistage et les campagnes de sensibilisation, elle sera amenée, sous peu, à une prise de décision cruciale, celle des fermetures des établissements éducatifs et par la suite la suggestion d'un enseignement en ligne afin de diminuer autant que possible les répercussions négatives. L'impact des crises et des urgences sur l'éducation au Liban s'est montré considérable et procède par étapes : de la prévention à la réaction et de l'adaptation à la transformation. Ainsi, il serait opportun d'effectuer une lecture ergonomique de la crise de la COVID-19 et de son influence sur l'enseignement à partir de la logique de la gestion de crises ou crisis management (Libaert, 2018). En effet, l'étymologie latine du mot «crise» emprunté au grec krisis montre la corrélation entre « décision » et «jugement $»$ (Le Robert, 1994) ; ce qui suppose un rapport de cause à effet, ou une conduite opérationnelle qui nécessite, au-delà de la communication, une prise de décision rapide et efficace, particulièrement au niveau éducatif. Dans son «Éducation COVID-19 : Cadre pour la planification de contingences, réduction des risques, préparation et réponse», l'Unicef adopte le modèle d'un 
«arbre de décision de réponse ${ }^{2}$ (Unicef, 2020) appliqué au contexte de l'apprentissage à distance durant la COVID-19. Bien qu'il soit conçu pour l'apprentissage scolaire, l'arbre est facilement transposable au contexte universitaire. Dans la figure 1, nous proposons une adaptation de l'arbre tel que conçu par l'Unicef au cadre académique libanais :

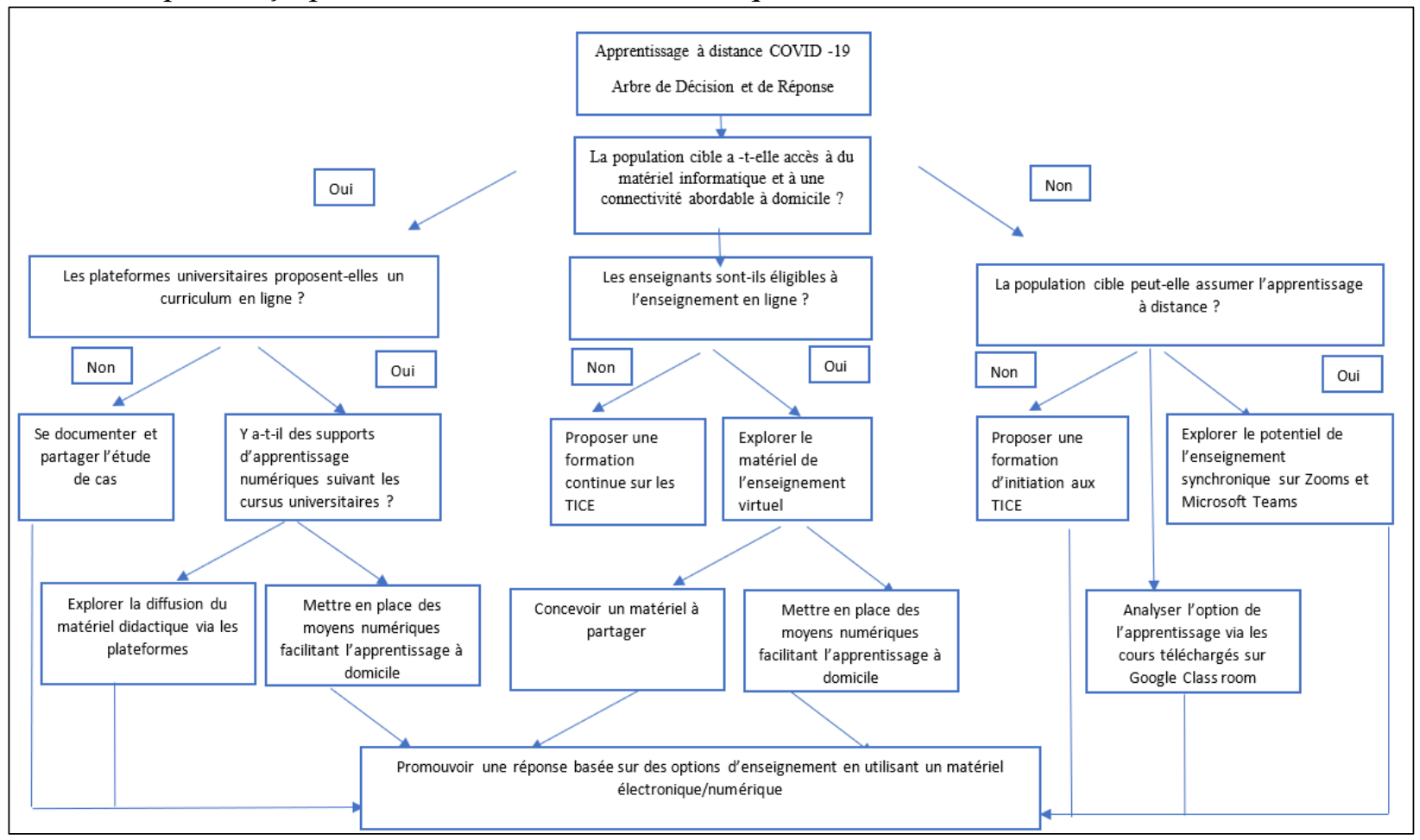

Figure 1 : Arbre de Décision de Réponse, enseignement/apprentissage à distance COVID-19

À partir de la figure 1, nous remarquons que trois axes régissent cet arbre de décision de réponse : les instances universitaires, les enseignants et les apprenants.

En ce qui concerne les universités, elles sont invitées à mettre en place des outils numériques pour faciliter aux enseignants et aux étudiants l'expérience de l'enseignement/apprentissage à distance et pour leur proposer des formations ponctuelles et professionnalisantes aux TIC selon le besoin. Les enseignants doivent, pour leur part, se former aux TIC et explorer le potentiel que ces dernières leur offre. Ils peuvent, par exemple, partager en ligne un matériel didactique ou mettre en place des cours du type co-modal. Les étudiants, quant à eux, seraient invités à maîtriser la manipulation des

${ }^{2}$ Ce document complète celui consacré aux « Messages clés et actions pour la prévention et le contrôle de la COVID-19 dans Unicef. (Mars 2020). Messages clés et actions pour la prévention et le contrôle de la COVID-19 dans les écoles. p.16. 
plateformes adoptées par les universités, telles que Google class room, Zoom et Microsoft Teams afin de rendre plus ergonomique le suivi des cours virtuels.

Dans cette perspective, tous les agents de l'acte de l'enseignement/apprentissage collaborent pour promouvoir une offre de cours basée sur des options éducatives virtuelles en utilisant un matériel numérique adéquat.

\section{Les TIC et les méthodes pédagogiques à l'université}

2.1. La maîtrise des TIC : une compétence de l'enseignant-universitaire

Depuis le début des années 2000, les référentiels du métier de l'enseignement supérieur imposent à l'enseignant-chercheur d'acquérir un savoir-faire devenu désormais indispensable à l'exercice de sa profession : la maîtrise des TIC.

Pour les auteurs des «10 compétences de l'enseignant universitaire», la compétence technologique représente un acquis inhérent au profil de tout enseignant-chercheur et renvoie à la quatrième compétence de leur référentiel « [m]aîtriser les diverses formes de la communication pédagogique» (Cahiers de l'IPM Belgique ${ }^{3}$, 2005). Avec l'évolution rapide des technologies, l'enseignant universitaire peut se rendre compte que les TIC peuvent influencer la qualité académique de son enseignement et lui permettre de développer des contenus et des méthodologies spécifiques. Cependant, si « la compétence technologique réfère au traitement de l'information, à la communication et à la collaboration [...] », elle implique, selon Brassard (2012) «la capacité d'un enseignant à agir dans un contexte de décloisonnement spatiotemporel de la classe ». Désormais, cette compétence «technologique » se présente comme une condition inhérente au profil des " enseignants-chercheurs nouvellement recrutés ${ }^{4}$ », au Québec, par exemple, ou en Algérie $^{5}$ et en France; cette dernière exige de "faire un usage professionnel des technologies de l'information et de la communication dans le respect des dispositifs législatifs et réglementaires en vigueur ${ }^{6}{ }$ ” (MESRI France, 2018). D'ailleurs, l'utilisation du numérique dans l'enseignement/apprentissage renforce la motivation chez l'apprenant et modifie profondément, selon Klein (2013), «les stratégies des élèves pour

3 Institut de Pédagogie universitaire et des Multimédias de l'Université Catholique de Louvain-la-Neuve.

${ }^{4}$ Ministère de l'Éducation et de l'Enseignement supérieur québécois. (2019). Cadre de référence de la compétence numérique.

${ }^{5}$ République Algérienne Démocratique et Populaire Ministère de l'Enseignement Supérieur et de la Recherche Scientifique. (2016). Référentiel de compétences des enseignantschercheurs nouvellement recrutés.

${ }^{6}$ Syndicat national de l'enseignement supérieur, Fédération syndicale unitaire. (2018). Référentiel métier des enseignants chercheurs, Document de travail du MESRI en France Version du 11 avril 2018, soumise à concertation. 
apprendre et des professeurs pour faire apprendre ${ }^{7}$ ». Dès lors, enseigner à distance ne se réduit pas à enregistrer des cours magistraux visualisés en ligne : il nécessite plutôt une conception des modules d'apprentissage personnalisés, des exercices en ligne et des évaluations à distance.

\subsection{L'intégration des TIC dans l'enseignement supérieur libanais}

Au Liban, au niveau des établissements universitaires, de grandes disparités ont été constatées dans l'utilisation des TIC. En effet, et en dépit de la pandémie, le secteur universitaire privé a intégré les TIC en son sein pour faire face aux exigences de la société et pour répondre aux besoins des apprenants (par exemple l'utilisation de la plateforme Moodle par les enseignants de l'Université de Balamand à partir de mars 2019, l'utilisation de l'application Microsoft Teams à l'Université Libanaise et l'Université Saint-Joseph (USJ) en mars 2019). Quant au secteur public qui dépend des investissements majoritairement publics, l'évolution est différente et l'inertie est plus forte.

En 2018, Zeitoun et Ghosn (2018) proposent un état des lieux de l'utilisation des TIC au Centre des Sciences du Langage et de la Communication $\left(\mathrm{CSLC}^{8}\right)$, rattaché à la Faculté des Lettres et Sciences Humaines à L'Université Libanaise. Les statistiques ont montré que 32\% des enseignants du CSLC n'intègrent pas les TIC dans leur enseignement mais $81 \%$ maitrisent les méthodes modernes d'évaluation. Le référentiel de compétences proposé, à cet égard, consacre une place particulière à la «compétence technologique» qui peut apporter une valeur ajoutée au processus d'enseignement/apprentissage allant de l'animation de la classe, à l'évaluation, du choix des stratégies efficaces au respect de la déontologie professionnelle et à la résolution des problèmes technologiques. Cependant ces indicateurs avancés ne fournissent pas de pistes pragmatiques pour la mise en œuvre de la compétence technologique dans l'enseignement/apprentissage, notamment dans un contexte d'enseignement hybride ou même en présentiel. « Le guide pour l'apprentissage et l'enseignement en ligne : le contexte de la pandémie Coronavirus » (Erasmus+, HERE, 2020) tente de combler cette « lacune » en présentant un diagnostic adapté au périmètre libanais. L'équipe Erasmus ${ }^{+}$propose d'intervenir auprès des universités du pays ${ }^{9}$, dans la mesure

${ }^{7}$ Klein, C. (2013). Les usages du numérique pour l'enseignement du FLE/FLS/FLSCO. L'école numérique, (16, juin 2013), 8-11.

${ }^{8}$ Selon les autrices, c'est l'un des «Centres les plus avancés dans la recherche et les formations qui mettent la technologie au service de l'enseignement, ce qui lui permet d'élargir les horizons actuels dans le domaine, et de tracer des perspectives d'avenir. Les diplômes fournis sont basés sur la technologie tel « Ingénierie de l'Apprentissage des Langues (IAL) » et Traitement Automatique des Langues (TAL)»

${ }^{9}$ Nous mettrons surtout l'accent sur la place accordée à la compétence technologique dans le document. 
où, la COVID-19 a contraint les universités à assurer une continuité pédagogique par le biais de l'enseignement virtuel. Les compétences en lien avec les TIC sont mises en place en un temps record et sans formations adaptées. C'est, dans cette perspective, que le guide susmentionné entend aider ces professionnels de l'enseignement. Les experts d'Erasmus+ remarquent la disparité (Erasmus+, HERE, 2020) entre les enseignants du supérieur, toutes spécialités confondues, quant à la maîtrise des outils technologiques. Cette constatation a été relevée auparavant par la recherche de Messai-Farkh et Kayal (2019) qui met en évidence cette intégration hétérogène des TIC dans leurs pratiques d'enseignement au sein de l'Université Libanaise. En se référant au Modèle de Raby (2004), ces chercheurs rapportent que «les enseignants (toutes disciplines confondues) sont majoritairement des utilisateurs intermédiaires des TIC. Un tiers des enseignants affirment ne pas les utiliser et $19 \%$ sont experts. Enfin, une minorité des enseignants est novice $(4 \%) »$.

Ainsi, il s'avère incontestablement indispensable de développer chez l'enseignant une série de compétences en TIC, utiles au nouveau cadre d'enseignement. À partir de ce constat, nous tenterons d'analyser l'impact de la pandémie sur l'enseignement de la langue française au sein des universités libanaises.

\section{Méthodologie de travail}

Un an après l'apparition de la COVID-19, une mise au point concernant la transition de l'enseignement en présentiel au virtuel s'impose. L'objectif serait de proposer aux institutionnels et aux enseignants de français $\mathrm{du}$ supérieur un meilleur encadrement par le biais d'une ingénierie pédagogique sur mesure et d'un plan de formation efficace.

\subsection{Participants et procédure}

Par souci de généralisation et d'objectivité, nous avons choisi de nous adresser à l'ensemble des enseignants de français exerçant au sein des universités privées et publique sur tout le territoire libanais. Afin de collecter des données suffisamment riches, nous avons utilisé conjointement une méthode d'analyse quantitative (enquête par questionnaire) puis une méthode d'analyse qualitative (entretiens individuels semi-directifs). Cette méthodologie mixte permet de développer une compréhension plus fine et plus riche du phénomène étudié recouvrant à la fois les aspects descriptifs et explicatifs de l'intégration des TIC dans l'enseignement en temps de COVID19. 


\subsection{Outils d'investigation}

L'enquête par questionnaire proposée cherche à mettre en évidence les représentations des enseignants par rapport à l'enseignement virtuel et à mieux comprendre les défis et les obstacles auxquels ils ont dû faire face tout au long de l'année écoulée.

\section{- L'enquête quantitative}

Notre enquête s'adresse à une population constituée de 110 enseignants de la langue française. Le questionnaire a porté sur la maîtrise des enquêtés en termes d'utilisation des TIC, des difficultés rencontrées durant leur expérience de l'enseignement à distance et des besoins ressentis pour mener au mieux l'enseignement virtuel. Seuls 30/110 des sondés ont répondu à notre questionnaire. Cela rend nos résultats relativement fiables du fait qu'ils représentent le tiers de la population étudiée et nous permettent de dresser un profil spécifique pour notre cohorte. Certains verbatims sont rapportés en adoptant le code suivant (E1) pour le premier entretien et ainsi de suite.

\section{- L'enquête qualitative}

Tout d'abord, nous avons proposé une enquête sous forme d'entretien individuel semi-directif avec 15 enseignants pour cerner les enjeux du passage à l'enseignement à distance en temps de pandémie. De plus, nous avons collecté les entretiens menés par le quotidien libanais l'Orient-le-jour (Eddé, 2020) et les témoignages transcrits d'enseignants de français partagés sous forme de vidéos le 26 novembre 2020, durant la « Journée internationale du professeur de français ». Ces résultats permettraient d'abord de retenir les obstacles rencontrés par les enquêtés et de déterminer ensuite leurs besoins en matière de formations.

\section{3. $\quad$ Traitement des données}

Le logiciel Statistical Package for the Social Sciences (SPSS) est utilisé dans le processus d'analyse des résultats assurant par-là la comparaison entre différentes entrées.

\section{Résultats}

\subsection{Profil de l'échantillon}

L'enquête quantitative montre que l'enseignement de la langue française reste au Liban l'apanage du genre féminin, du fait que 27/30 des enseignants enquêtés sont des femmes. Toutes les personnes ont un contrat à l'Université Libanaise (UL) ${ }^{10}$, et $3 / 30$ seulement d'entre elles ont un contrat parallèle dans une ou parfois deux universités privées. La majorité ${ }^{11}$ enseigne

\footnotetext{
${ }^{10}$ La seule université publique au Liban

${ }^{11} 56.7 \%$ pour le niveau A2 (soit $17 / 30$ ), $66.7 \%$ pour le niveau B1 (soit 20/30) et $60 \%$ pour le niveau B2 (soit 18/30).
} 
les niveaux A2 et B1 selon le Cadre Européen Commun de Référence pour les Langues (CECRL) et elle est en charge de cours dans pratiquement toutes les spécialités qu'offrent les différentes facultés. 14/30 enseignants de notre panel ont également des fonctions à responsabilités (coordination des cours, etc.). La moitié des professeurs enseigne depuis plus de 10 ans des cours de français inscrits dans le cursus. Cependant, 24/30 de notre panel dispensait des cours durant l'année académique 2019-2020. L'apparition de la COVID-19 et l'instauration des cours en distanciel ont obligé certaines universités (comme l'UL et l'USJ) à réduire les effectifs du personnel enseignant.

\subsection{Le rapport aux TIC}

Le rapport des protagonistes à la technologie est complexe : près de la moitié des enseignants affirme recourir aux $\mathrm{TIC}^{12}$ dans leur enseignement avant le confinement imposé en mars 2020. Cependant, 16/30 d'entre eux avouent que grâce à l'enseignement virtuel, ils ont pu suivre l'avancement et la compréhension des connaissances et des compétences que les technologies mettent en jeu. En effet, avant la pandémie, pour 12/30 de notre panel, l'utilisation professionnelle des TIC en relation avec les tâches académiques se réduisait essentiellement à la recherche documentaire. Avec le bouleversement imposé, 28/30 des enquêtés estiment s'être adaptés facilement et rapidement aux situations d'apprentissage en distanciel (même si environ le tiers des répondants estiment le faire relativement avec certaines difficultés) et 13/30 d'entre eux déclarent avoir rencontré quelques ennuis dans la gestion de la classe à distance.

${ }^{12}$ Comme les plateformes adoptées par la majorité des universités libanaises : Google class room, Zoom et Microsoft Teams. 


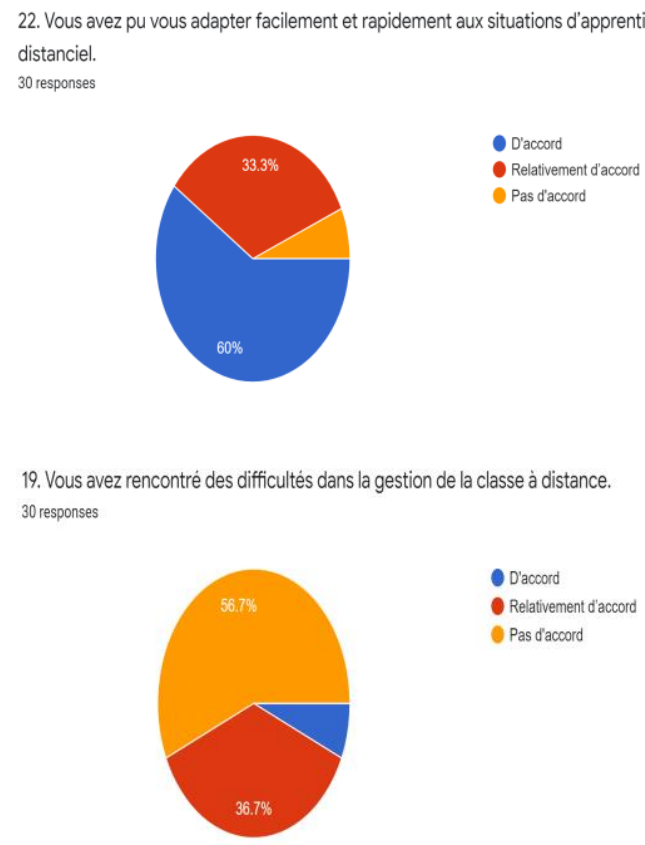

Figure 2 : Niveaux d'adaptation et de difficulté face aux situations d'apprentissage en distanciel

De ce fait, ce n'est pas l'aspect logistique ou l'acquisition des compétences numériques qui posent problème ; mais c'est la transposition en ligne de l'activité d'enseignement, à savoir l'interaction virtuelle. D'ailleurs les enseignants au Liban indiquent souffrir d'un manque de support technique (27/30), de l'absence de formation adéquate (9/30) ainsi que de l'insuffisance du soutien pédagogique $(8 / 30)$.

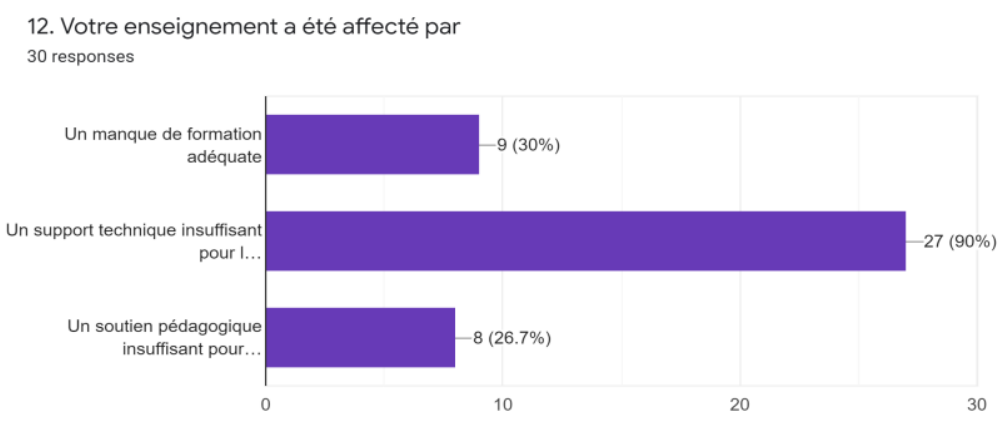

Figure 3 : Facteurs impactant l'enseignement en ligne

Ajoutons à cela, les problèmes techniques de connexion internet (26/30) et l'absence des équipements techniques appropriés chez les étudiants 
(18/30) qui affectent le processus de l'enseignement en ligne et entravent le travail de l'enseignant. Ce dernier trouve des difficultés (24/30) dans la recherche des ressources (contraintes administratives / budgétaires / technologiques).

14. Vous avez trouvé des difficultés dans la recherche des ressources (contraintes

administratives/budgétaires/technologiques)

30 responses

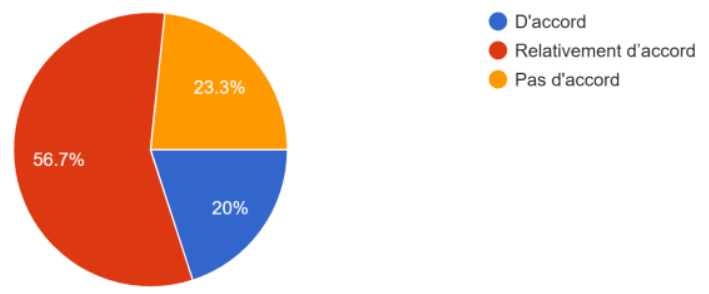

Figure 4 : Niveaux de difficulté dans la recherche des ressources

Quant aux outils pédagogiques, ils varient entre la visio-conférence, le chat, le forum (26/30) et le recours simultané au partage des fiches pédagogiques. Certes, la situation bouleversante de la COVID-19 a incité le renforcement des pratiques numériques des enseignants qui se trouvent dans l'obligation de s'adapter aux nouveaux contextes d'apprentissage. Cette transition est, selon les sondés (29/30) facilitée par les institutions ${ }^{13}$.

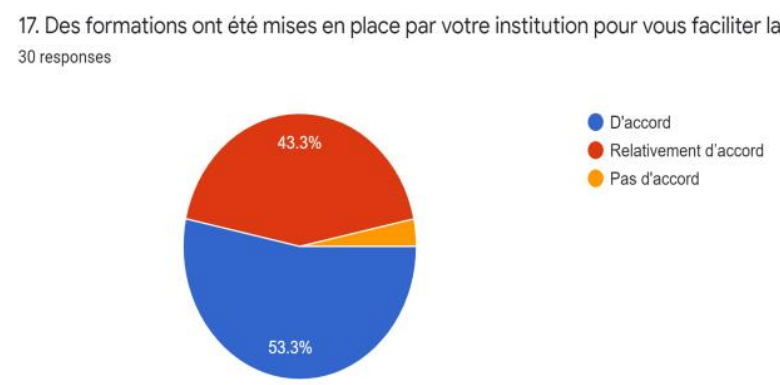

Figure 5 : Soutien pédagogique mis en place par les institutions

${ }^{13}$ À ce titre, l'Université Libanaise a lancé un lien-guide pour l'utilisation de Zoom et de Microsoft Teams. Parallèlement, l'USJ a organisé un programme de formation pour l'année 2020-2021 allant de la conception et la planification d'un enseignement en ligne, aux méthodes et techniques d'enseignement en ligne, à l'accompagnement des étudiants en ligne et enfin à l'évaluation des acquis des étudiants et la rétroaction en ligne. https://www.usj.edu.lb/inst/upload/7827e36286d11522287e2baa82d5d6c5.pdf 
Mais en même temps, il incombe aux enseignants de miser sur les activités interactives et le développement de la compétence interculturelle afin de maintenir la motivation de l'audience.

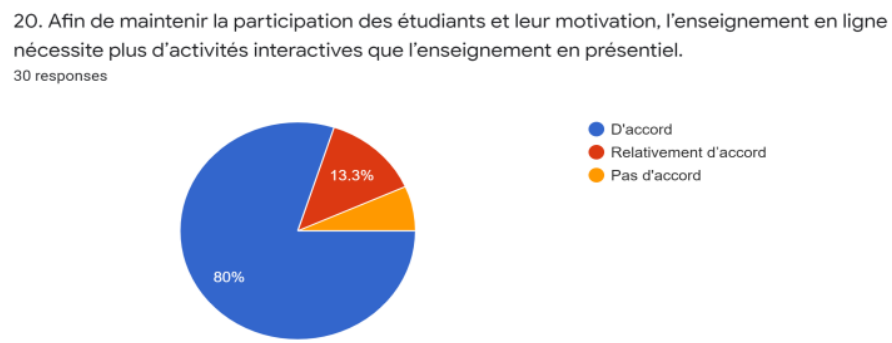

Figure 6 : nécessité d'adaptation des cours virtuels

\subsection{Impact de la COVID-19 sur l'enseignement/apprentissage du français}

En dépit des problèmes techniques constatés tels que le faible débit de connexion et des coupures d'électricité, 24/30 des enseignants confirment leur préférence pour un enseignement en ligne en temps de pandémie (figure 7). Dans un souci de prévention et de lutte contre le coronavirus, 15/30 des enquêtés ont mis en place des activités pédagogiques (lexicales ou thématiques) en lien avec le virus (figure 8). L'objectif est d'initier les étudiants aux problèmes de la santé, ou encore de les sensibiliser aux enjeux médicaux. Familiariser l'étudiant à la terminologie propre à la pandémie répond à un besoin spécifique et permet à tout un chacun d'éviter les erreurs liées à la méconnaissance qui entoure le coronavirus.

18. Malgré les problèmes de connexion et d'électricité, je préfère enseigner en ligne en temps

de Covid19.

30 responses
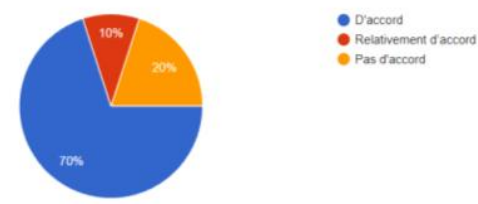

Figure 7 : préférence pour enseigner en ligne sous COVID-19
23. Avez-vous dejà mis en place des activités de langue en lien avec la Covid19?

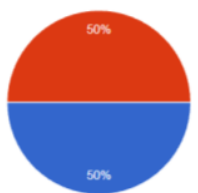

Figure 8 : activités pédagogiques mises en place en lien avec la pandémie

Ces différents exercices peuvent se réaliser par le biais de formations hybrides (15/30), de l'enseignement à distance (20/30) ou encore de l'exploitation du web social (17/30) (figure 9). 


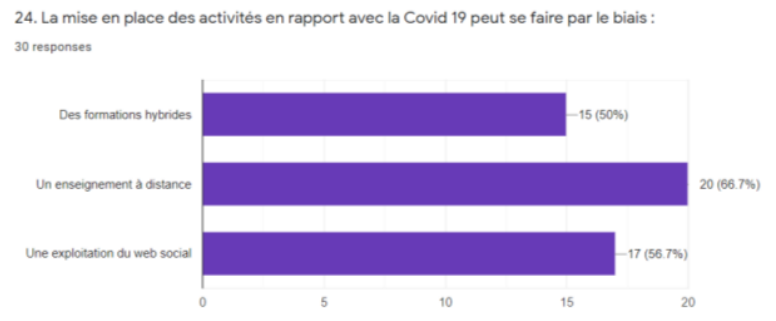

Figure 9 : moyens envisageables pour mettre en place des formations continues

\subsection{Besoins de formations ressentis par les enseignants}

Malgré cette volonté de croiser les problèmes sanitaires et les contenus d'enseignement, 18/30 de notre panel rencontrent des difficultés à aborder des sujets en lien avec la pandémie notamment dans les cours dispensés aux débutants (niveaux A1/A2 du CECR). Bien que notre cohorte soit composée de professionnels expérimentés dans le domaine de l'enseignement de la langue française à l'université, elle exprime (17/30) des besoins en terme de formations ; la réalité sanitaire inédite entraine naturellement une adaptation de leurs habitudes professionnelles. Les verbatims suivants traduisent leur volonté d'adapter l'enseignement de la langue française aux contextes environnants.

Tableau 1 : Verbatims

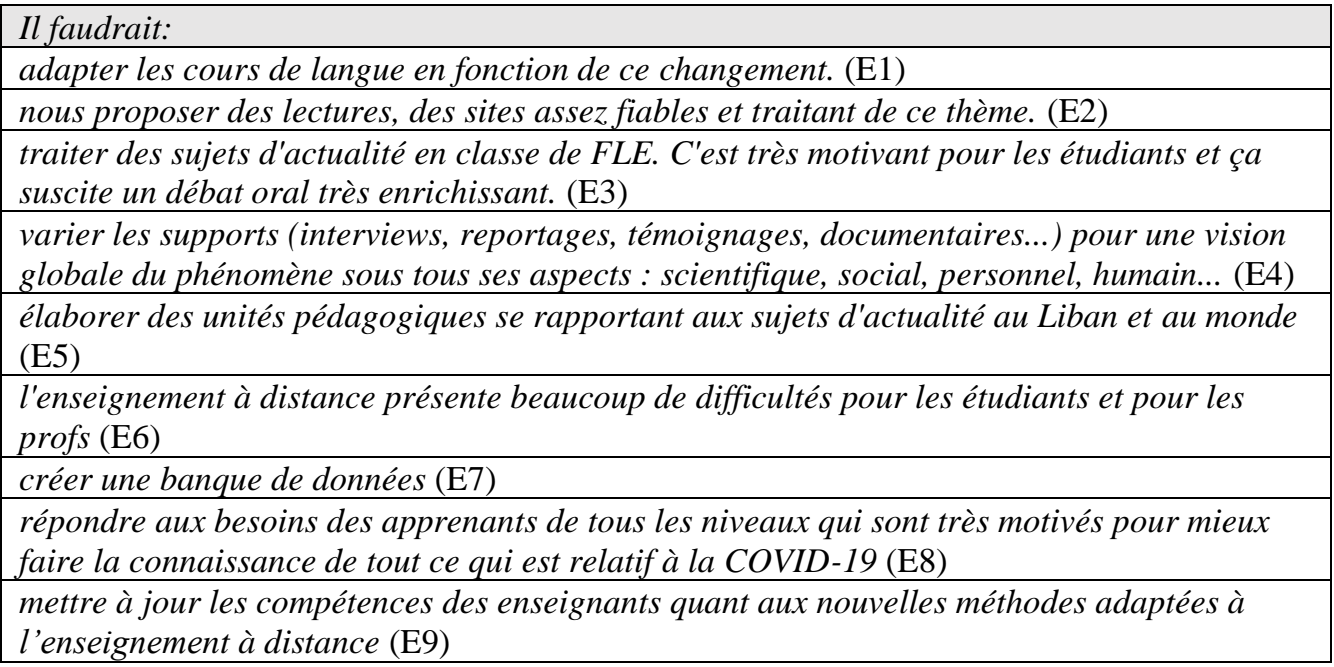

La moitié des enseignants n'a jamais utilisé les TIC avant le confinement (Figure 10) et se trouve ainsi tiraillée entre des priorités sanitaires et une profession qui devient de plus en plus exigeante. 


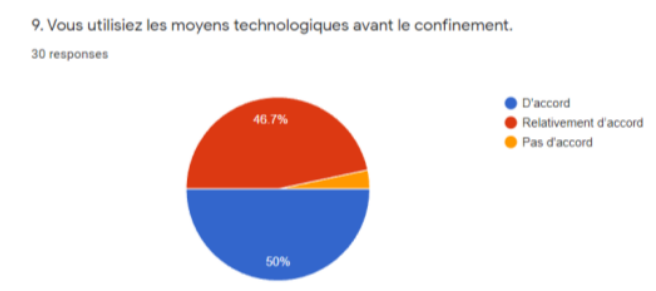

Figure 10 : utilisation des TIC avant le confinement

C'est sans doute pour cette raison que 24/30 d'entre eux ont effectivement éprouvé des difficultés dans ce passage hâtif à l'enseignement virtue $1^{14}$ et ont affirmé que « l'enseignement à distance présente beaucoup de difficultés pour les étudiants et pour les profs »(E6). Certains ont même demandé explicitement à leur institution de « mieux former les enseignants et les étudiants afin d'améliorer la qualité de l'enseignement à distance durant la période de confinement à cause de la pandémie ». D'où l'urgence de professionnaliser les enseignants et de redéfinir les compétences professionnelles.

\subsection{Désir de développer ses compétences}

La Journée Internationale du Professeur de Français (le 26 novembre 2020) nous a permis de recueillir des données à partir des témoignages des professeurs qui ont suivi une série de formations ${ }^{15}$ en lien avec l'enseignement en ligne, les outils de conception numérique, l'évaluation ou les techniques d'animation des cours et des groupes.

Certains soulignent l'utilité de l'enseignement en ligne dans la mesure où il procure un accès facile et rapide à l'information pour l'étudiant et pour le professeur. Malgré la distance physique et sociale imposée, les enseignants ont témoigné d'une ambiance solidaire et d'entraide entre eux, leurs étudiants et leurs collègues. Mais un des enseignants du groupe se trouvait face à un défi majeur tel que "savoir gérer le cours en ligne », en matière de temps et d'interaction surtout que la numérisation des contenus s'imposait comme une nécessité et supposait une conception de préparation spécifique qui révolutionne l'enseignement/apprentissage traditionnel. À cet égard, nous surlignons les propos suivants d'une enseignante : «l'essor de l'enseignement à distance, qui est survenu à l'ère de la COVID-19, survivra très probablement à la crise sanitaire et persistera pour laisser un impact sur tout le domaine de l'éducation dans le monde et durant les années à venir »; et une autre enseignante de continuer: "même si la crise sanitaire disparait, un apprentissage mixte n'est pas une mauvaise idée ». Certes il y a approbation

\footnotetext{
${ }^{14} \mathrm{Cf}$. la description détaillée de la situation au Maroc présentée par Naciri, K. (11 août 2020).

${ }^{15}$ Pour sa part, le Bureau des Langues de l'Université Libanaise a proposé une série d'actions sur l'enseignement en ligne à laquelle les enseignants de français ont pu participer.
} 
sur cet aspect révolutionnaire, cependant, subsiste le souci de l'évaluation « des différentes compétences langagières de l'oral et de l'écrit en ligne, surtout que l'évaluation des langues diffère [...] de celle des disciplines scientifiques où l'on peut recourir à des questions du type QCS ou QRM », souligne une collègue.

Malgré leur solide expérience, les enseignants expriment leur réticence et ne semblent pas maîtriser les enjeux de l'enseignement à distance. En témoignent les propos suivants : «J'ai tenté de faire de mon mieux pour utiliser une approche pédagogique efficace à l'enseignement à distance et de varier les modalités de travail en fonction des situations : cours magistral, discussions en groupes, travail en plénière. C'est en quoi consiste mon travail actuellement en ligne $»$. Cet embrouillement permet de confirmer l'hypothèse $\mathrm{du}$ besoin de former les enseignants pour que l'enseignement en ligne ne se transforme pas en une simple copie virtuelle du cours donné de manière « traditionnelle».

En somme, selon Cuban (1999), Karensti et al (2004) «pour certains gouvernements, les obstacles liés à l'intégration des TIC se limiteraient à trois facteurs : l'équipement ou les logiciels, le temps et le soutien technique ». Un investissement majeur dans ces trois domaines devrait permettre, selon les auteurs, de favoriser l'intégration pédagogique des TIC en éducation. Or, nos résultats mettent clairement en exergue que les facteurs psychologiques (générer une motivation dans le processus d'enseignement/apprentissage), sociaux (assurer une vision globale de la pandémie sous tous ses aspects : scientifique, social, personnel, humain...), idéologiques (développer de nouvelles pratiques enseignantes pour accompagner les changements sociaux), organisationnels (encadrer les enseignants et leur proposer un ensemble de formations en continu (ex. de l'USJ).) et institutionnels (rôle du gouvernement, du Ministère de l'éducation, des recteurs des universités, etc.) sont aussi importants que les facteurs classiques tels que le temps, l'argent (ressources matérielles) dans l'intégration des TIC à la pratique éducative des enseignants et à l'innovation techno-pédagogique.

\section{Analyse et recommandations}

Les enquêtes qualitatives et quantitatives réalisées en amont nous amènent à apporter une série de recommandations. Nous exposons tout d'abord le bilan de l'enseignement en ligne du français sous COVID-19.

\subsection{Bilan de l'enseignement en ligne du français sous COVID-19}

Depuis mars 2019, les universités ont passé à l'enseignement à distance. La transition n'était pas simple vu l'absence d'une infrastructure technologique et de professeurs solidement formés. Les coupures du courant électrique, le mauvais débit d'internet, le manque de fondement 
technologique, la situation gravissime au Liban due aux crises sanitaire et économique, sont autant de facteurs qui empêchent le gouvernement de concevoir « un arbre de décision » adéquat pour rendre optimale la transition de l'enseignement en présentiel au virtuel et pour dissiper les obstacles en termes de continuité pédagogique dans le supérieur. Les enseignants ${ }^{16}$ montrent, dès lors, leur volonté de s'adapter aux modalités de l'enseignement virtuel même s'ils jugent que leur formation initiale et continue ne leur a pas permis d'acquérir ou de développer des compétences technologiques. Ce qui est sûr, c'est que, même avec l'impact vital des crises au Liban, les enseignants restent soucieux de leurs acquis et veulent à tout prix évoluer pour une meilleure performance. La Journée Internationale du Professeur de Français constitue une sorte de bilan d'expériences pour les enseignants de français : « le professionnel se construit à partir de ses expériences mais aussi grâce à sa capacité à penser l'action. La capacité à prendre du recul est nécessaire à l'action » comme le stipulent Schön (1983) et Longuet et Springer (2012). Cette prise de recul leur a permis, d'une part, de comprendre que la relation entre le formateur et l'apprenant est devenue multidirectionnelle (du formateur à l'apprenant / de l'apprenant au formateur / de l'apprenant à lui-même) ; d'autre part, ils ont eu l'occasion d'examiner les différents savoirs exploités dans l'enseignement virtuel et d'envisager, par la suite, des remédiations possibles à leurs pratiques enseignantes. Ci-dessous, un tableau récapitulatif des différentes habiletés investies :

Tableau 2 : Habiletés réellement investies par les enseignants de français sous COVID-19

\begin{tabular}{|l|l|l|}
\hline \multicolumn{1}{|c|}{ Savoirs } & \multicolumn{1}{|c|}{ Savoir-faire } & \multicolumn{1}{c|}{ Savoir-être } \\
\hline $\begin{array}{l}\text { Maîtriser le contenu des } \\
\text { plateformes qui offrent des } \\
\text { formations en ligne (MOOC } \\
\text { ou portails ouverts). }\end{array}$ & $\begin{array}{l}\text { Être capable de concevoir des cours } \\
\text { adaptés à l'enseignement en ligne. }\end{array}$ & $\begin{array}{l}\text { Être capable de remettre en question } \\
\text { son cours et de l'adapter en fonction } \\
\text { des besoins des apprenants. }\end{array}$ \\
\hline $\begin{array}{l}\text { Maîtriser les stratégies } \\
\text { d'enseignement. }\end{array}$ & $\begin{array}{l}\text { Être capable de préparer/former ses } \\
\text { étudiants à l'enseignement en ligne. }\end{array}$ & $\begin{array}{l}\text { Etre capable de briser la glace / la } \\
\text { distance qui se crée en ligne. }\end{array}$ \\
\hline $\begin{array}{l}\text { Maîtriser les méthodes } \\
\text { d'enseignement. }\end{array}$ & $\begin{array}{l}\text { Être capable d'acquérir des stratégies } \\
\text { d'autorégulation, des compétences en } \\
\text { matière de gestion du temps. }\end{array}$ & $\begin{array}{l}\text { Être capable de bien communiquer } \\
\text { virtuellement. }\end{array}$ \\
\hline Maîtriser les TIC. & $\begin{array}{l}\text { Faire preuve d'une bonne } \\
\text { gestion/organisation. }\end{array}$ & $\begin{array}{l}\text { Être capable de diversifier les } \\
\text { stratégies d'enseignement. }\end{array}$ \\
\hline $\begin{array}{l}\text { Maîtriser les fondements de la } \\
\text { classe renversée. }\end{array}$ & $\begin{array}{l}\text { Être capable de construire une } \\
\text { communauté estudiantine en ligne. }\end{array}$ & $\begin{array}{l}\text { Être réactif et adapter le contenu de } \\
\text { son cours. }\end{array}$ \\
\hline
\end{tabular}

Le tableau 2 conçu, suite à une collecte des témoignages des entretiens menés à la fois lors de la «Journée internationale du professeur de français »

${ }^{16}$ Cité par Jarraud, F. (27 novembre 2020). Philippe Meirieu : Enseigner : un métier de résistant. L'Express. Café pédagogique. Lien :

http://www.cafepedagogique.net/lexpresso/Pages/2020/11/27112020Article6374205755276

75818.aspx 
et par le quotidien libanais l'Orient-le-jour (Eddé, 2020), met en évidence la mise en place des habiletés sans formation préalable. Les enseignants n'ont pas cédé et ont décidé de relever le pari en optant pour des formations qui leur garantissent professionnalisme et perfectionnement, ce qui n'est pas sans rappeler le concept d'autoformation, définie par Tremblay (2003) comme étant " une situation pendant laquelle l'individu est responsable de l'une ou l'autre dimension d'une activité de formation : contenu, objectifs, démarche, ressources, rythme ». Dans cet esprit, les enseignants revendiquent le besoin d'une plateforme accessible où il serait loisible de partager expériences, craintes et attentes, mais aussi documents et cours. L'idéal serait de générer une solidarité entre collègues pour construire ensemble le nouveau profil qu'impose la situation à travers le monde, celui d'un enseignant "technicien porteur de services »(Jarraud, 2020). Certes, la nécessité de formations s'impose. Soucieux d'assurer un encadrement professionnel, il convient de réitérer les propos de Zeitoun et Ghosn (2018) et de proposer une formation adaptée; elle devrait être focalisée, à notre sens, sur l'enseignement du français virtuel et de la COVID-19 et de sa représentation dans le domaine de l'art visuel. Cette entrée serait innovatrice et s'intègrerait pleinement au quotidien des apprenants.

\subsection{Propositions didactiques en lien avec COVID-19}

Développer les compétences numériques et les investir, par le biais de l'art, dans un travail de conception de curricula en rapport avec la pandémie survenue, telle est notre intention. En effet, le support artistique constitue une base de communication qui, via le code visuel, maintient la motivation et facilite la compréhension et par suite, l'interaction entre apprenant et enseignant. L'art « facilite ainsi l'accès à la langue cible et permet un meilleur traitement cognitif et pratique des contenus disciplinaires ${ }^{17} »$. En effet, en qualité d'enseignants du supérieur, notre projet a pour ambition d'offrir de nouveaux moyens pour permettre d'atteindre les objectifs pédagogiques de l'enseignement du français à l'université. Il ne s'agit pas seulement de travailler sur un lexique spécifique mais aussi de mobiliser les compétences communicationnelles et langagières en lien avec la santé publique. La mise en situation pratique d'outils didactiques issus des arts visuels repose en effet sur une méthodologie qui relève à la fois d'une approche interculturelle de l'enseignement des langues et de la pédagogie de la tâche. Ainsi, nous partons de thématiques représentées dans des toiles en lien avec des pandémies survenues tout au long de l'histoire humaine et cherchons à les investir au compte de l'enseignement de la langue dans un contexte de COVID-19 (Gac,

${ }^{17}$ Witzigmann, S. (2014). L'art dans tous ses états, synergies entre le français langue étrangère et les arts plastiques, $\mathrm{N}^{\circ} 6,2014.5$ Editions du CRINI () e-crini. ISSN 1760-475. 
2007). Nous citons à titre d'exemple certains sujets comme l'étude des symptômes causés par la peste, la syphilis ou le choléra, l'équipement des centres médicaux ou l'installation d'hôpitaux de campagne durant la grippe espagnole, le recours aux traitements à base de plantes médicinales ou aux médicaments synthétiques pour les pandémies, les mesures du confinement, le masque du « Dr Schnabel », etc. Le choix des thématiques ${ }^{18}$ est large et cette entrée proposée par les œuvres d'art serait, à notre sens, un moyen culturel riche, profondément exploitable et une manière inédite pour aborder la pandémie dans un cours de langue. Dans cette perspective, nous avons entrepris un succinct recensement des œuvres d'art qui mettent en exergue des thématiques en lien avec le domaine médical et qui permettent à l'enseignant de travailler des tâches pédagogiques ciblées. Nous présentons dans le tableau 3 un matériel varié des œuvres d'art et des thématiques engagées

Tableau 3 : Propositions de matériels de cours en lien avec la COVID-19

\begin{tabular}{|c|c|c|c|c|}
\hline Problème soulevé & Artiste & Titre de l'œuvre & Année & $\begin{array}{l}\text { Thématique } \\
\text { engagée }\end{array}$ \\
\hline Les plantes médicinales & Anonyme & Dioscoride et son étudiant & 1229 & $\begin{array}{l}\text { Les traitements à } \\
\text { base de plantes } \\
\text { médicinales }\end{array}$ \\
\hline $\begin{array}{l}\text { La professionnalisation } \\
\text { du métier de } \\
\text { l'apothicaire }\end{array}$ & $\begin{array}{l}\text { Hieronymus } \\
\text { Brunschwig }\end{array}$ & $\begin{array}{l}\text { Représentation d'un maître } \\
\text { apothicaire donnant la leçon à } \\
\text { son apprenti, extrait de Das } \\
\text { Buch der Gesundheit (le livre } \\
\text { de la santé) }\end{array}$ & 1505 & $\begin{array}{l}\text { La naissance de } \\
\text { l'apothicairerie }\end{array}$ \\
\hline \multirow{3}{*}{ La peste } & $\begin{array}{c}\text { Josse } \\
\text { Lieferinxe }\end{array}$ & $\begin{array}{l}\text { Saint Sébastien intercède pour } \\
\text { les victimes de la peste }\end{array}$ & 1508 & $\begin{array}{l}\text { Le rapport à la mort } \\
\text { et l'impact social : } \\
\text { peur, panique, etc. }\end{array}$ \\
\hline & $\begin{array}{c}\text { Pieter } \\
\text { Bruegel } \\
\text { l'Ancient }\end{array}$ & Le triomphe de la mort & 1562 & $\begin{array}{c}\text { Le taux de mortalité } \\
\text { élevé / l'impact } \\
\text { social de la } \\
\text { pandémie }\end{array}$ \\
\hline & Anonyme & Docteur Schnabel / Dr Beak & vers 1656 & $\begin{array}{c}\text { Le masque en forme } \\
\text { de bec }\end{array}$ \\
\hline La syphilis & $\begin{array}{l}\text { Joaquin } \\
\text { Sorolla }\end{array}$ & Triste héritage & 1899 & Les symptômes \\
\hline \multirow[t]{2}{*}{ La grippe espagnole } & Anonyme & $\begin{array}{c}\text { Un hôpital italien surchargé } \\
\text { pendant l'épidémie de grippe } \\
\text { espagnole de 1918-1919. }\end{array}$ & \multirow[t]{2}{*}{1918} & $\begin{array}{l}\text { Les installations } \\
\text { hospitalières / Les } \\
\text { hôpitaux de }\end{array}$ \\
\hline & Egon Schiele & La famille & & campagne \\
\hline \multirow{3}{*}{ La COVID-19 } & Lutfi Jaafar & Le Coronavirus & \multirow{3}{*}{2020} & $\begin{array}{c}\text { La calligraphie arabe } \\
\text { en lien avec la } \\
\text { pandémie }\end{array}$ \\
\hline & Leila Dagher & $\begin{array}{c}\text { Social distancing / Tribute to } \\
\text { Heroes }\end{array}$ & & Le masque préventif \\
\hline & Jessy Tabet & Ville fantôme & & Le confinement \\
\hline
\end{tabular}

${ }^{18} \mathrm{cf}$. Haddad, G. (30 octobre, 2020). Epidemics and the Arts. 
Le tableau 3 met en relief la dimension culturelle de notre recherche. C'est un terrain prometteur pour toute personne qui compte s'y investir, mais une formation à la conception des outils didactiques est nécessaire avant d'entamer ce type d'enseignement.

Pour expliciter davantage nos propos nous citerons deux exemples qui pourraient servir de matériels pédagogiques importants. Le premier est tiré du neuvième art : la bande dessinée. Une aventure 100\% belge! Les internautes ont adapté la série de Tintin en mode pandémie comme « Le focus blues », «Les Dupondt ont chopé le COVID », «Tintin et le Coronavirus », «Le spectre du virus », « Haddock pète les plombs en confinement», « Tintin a oublié son attestation» (Renassia, 2020). Cet exemple pourrait être exploité pour investir, avec une dose d'humour, l'impact psychologique du confinement sur la société ou pour expliquer les mesures de sécurité ou de précaution imposées par les gouvernements ou la communauté médicale.

Le second exemple choisi nous renvoie au « street $\operatorname{art}^{19}{ }^{19}$. En effet, une vague d'artistes et d'écrivains décident d'adapter des toiles connues au contexte de la pandémie : des peintures murales et des graffitis remplissent les rues et des installations murales de tout genre transforment les villes. Cet exemple est sans doute le plus riche d'entre tous car, depuis le confinement, les artistes du monde entier ont fait porter le masque, chacun à sa manière, à des personnages du domaine politique, artistique ou social. Ghiath Al Robih de Art of change a même créé une version dédiée au corps médical libanais et \#bradytheblack leur a consacré sur son compte Instagram une série de « Corona sketches ». Par ailleurs, des artistes comme Leila Dagher ou Jessy Tabet ont choisi de représenter le véritable impact de la pandémie sur la société libanaise. La première peint la solitude et la désolation, la distanciation sociale ou encore le danger qui hante l'espèce humaine ${ }^{20}$. La seconde voit l'aprèsmort ou l'apocalypse dans sa «Ville fantôme ». Même la calligraphie arabe n'est pas restée indifférente. « Le coronavirus » de Lutfi Jaafar exprime avec beaucoup de scientificité la constitution du virus de cette nouvelle pandémie. En somme, nous pouvons affirmer que notre proposition peut se décliner en autant de propositions appropriées aux différentes spécialités des apprenants. Il est évident que la COVID-19 a touché toutes les tranches d'âge, toutes les classes sociales et tous les domaines. Dans ce sens, elle peut être exploitée dans toutes les spécialités en mettant à chaque fois l'accent sur l'intérêt et les

19 Mitman, T. (18 mai 2020). Coronavirus murals: inside the world of pandemic inspired street art. Ricci, B. (2020). Coronavirus Street Art: How The Pandemic Is Changing Our Cities. Erdekian, A. (26 mai 2020). The Coronavirus Street Art Helping Lift Spirits Around the World.

${ }^{20}$ Cf. les peintures de Leila Dagher: «Endagered specie», «Lockdown», « Social distancing », « Tribute to heros, Confined » et « Village lockdown ». 
besoins langagiers des apprenants en question. Dans le tableau 4, nous présentons une série de propositions adaptées aux facultés :

Tableau 4 : Propositions de thématiques en lien avec la COVID-19:

\begin{tabular}{|c|l|}
\hline Faculté(s) & \multicolumn{1}{|c|}{ Thématique(s) } \\
\hline $\begin{array}{c}\text { Sciences Médicales, Médecine } \\
\text { Dentaire, Pharmacie et Santé } \\
\text { Publique }\end{array}$ & $\begin{array}{l}\text { Recherches médicales sur la COVID-19 ; Mutation du } \\
\text { Coronavirus ; Problématique de la vaccination ; Soutien } \\
\text { psychologique pour les personnes en confinement... }\end{array}$ \\
\hline $\begin{array}{c}\text { Lettres et Sciences Humaines et } \\
\text { Beaux-Arts et Architecture }\end{array}$ & $\begin{array}{l}\text { Impact du confinement sur les arts : Fruits confits (ateliers } \\
\text { d'écriture : USJ-UTP) ; \#artenquarantaine ; Opéra en ligne ; } \\
\text { Graffitis... }\end{array}$ \\
\hline Gestion & $\begin{array}{l}\text { Dépression des étudiants ; Gestion de crises sanitaires ; } \\
\text { Installation d'hôpitaux de campagne ; Engouement pour } \\
\text { l'achat en ligne et développement des sites de vente en ligne ; } \\
\text { E-commerce }\end{array}$ \\
\hline $\begin{array}{c}\text { Droit et Sciences Politiques et } \\
\text { Administratives }\end{array}$ & $\begin{array}{l}\text { Imposition du couvre-feu ; Nouvelle règlementation du droit } \\
\text { de travail (travail à la maison versus travail en entreprise) }\end{array}$ \\
\hline Génie et Technologie & $\begin{array}{l}\text { Mise en place d'une infrastructure technologique qui assure de } \\
\text { bonnes conditions pour l'enseignement/apprentissage en ligne } \\
\text { et pour le télétravail... }\end{array}$ \\
\hline Sciences & $\begin{array}{l}\text { Invention de robots utilisés auprès de personnes atteintes de } \\
\text { COVID-19; Analyse des PCR (UL) }\end{array}$ \\
\hline Pédagogie & $\begin{array}{l}\text { Défi de l'enseignement à distance ; Décrochage scolaire- } \\
\text { universitaire ; Sensibilisation de toute la population au sujet de } \\
\text { la COVID-19... }\end{array}$ \\
\hline Tourisme et gestion hôtelière & $\begin{array}{l}\text { Transformation des hôtels en hôpitaux de quarantaine ; } \\
\text { Installation médicale à l'aéroport (tests PCR, etc.) }\end{array}$ \\
\hline Campagne d'éveil et d'éducation sur la COVID-19... \\
\hline
\end{tabular}

Afin d'y apporter une orientation pragmatique, nous proposons une fiche pré-pédagogique à finaliser et expérimenter lors des nouvelles formations. Elle comprend une exploitation pédagogique de différents supports authentiques issus du terrain en rapport avec la COVID-19 et les œuvres d'art. Cette fiche amène l'étudiant inscrit dans les facultés des sciences médicales et de santé publique à développer sa faculté d'analyse pour exprimer sa satisfaction à propos des services médicaux dispensés dans les hôpitaux de campagne. Les activités choisies forment un parcours d'apprentissage et visent la réalisation de la tâche suivante : écrire une lettre officielle au Ministre de la Santé pour optimiser la qualité des soins et promouvoir les hôpitaux de campagne en temps de COVID-19. Tout d'abord, nous proposons aux étudiants de travailler sur un document de compréhension orale à partir duquel l'étudiant se familiarise avec le thème des hôpitaux de campagne. Il sera amené à répondre à des questions via la plateforme Edpuzzle. Vient ensuite un travail de compréhension écrite à partir de la plateforme TEDEd et/ou Google Forms. Cette activité permettra de s'assurer de la compréhension de l'enjeu de l'installation des hôpitaux de campagne. Ensuite, en prenant comme support de travail des extraits des documents audiovisuel et écrit déjà travaillés 
à l'étape précédente, les activités de repérage permettront d'identifier, entre autres, le lexique médical en lien avec la COVID-19 ${ }^{21}$. Les étudiants pourront également procéder à un repérage des expressions de la cause, de la concession et de la comparaison.

Enfin, l'ultime travail consiste à exécuter la tâche finale. Elle se fera sous tutelle de l'enseignant de français qui accompagnera les étudiants à échanger à l'oral et à l'écrit au sujet des hôpitaux de campagne. Ces échanges seront initiés par des supports artistiques. Les activités pourront être travaillées à partir de Microsoft Teams ou Zoom et une mise au point avec l'enseignant de français s'effectuera via les plateformes susmentionnées. Ainsi, l'étudiant pourra aborder la tâche finale grâce aux différents échanges et commentaires apportés.

${ }^{21}$ Cf. Bureau du traducteur, 11 juin 2020, Lexique sur la pandémie de COVID-19. 
Tableau 5 : Fiche pré-pédagogique

\section{FICHE PRE-PEDAGOGIQUE}

\begin{tabular}{|c|c|c|c|}
\hline Thème & \multicolumn{3}{|c|}{ Les hôpitaux de campagne en temps de COVID-19 } \\
\hline Public & \multicolumn{3}{|c|}{ Etudiants inscrits aux facultés médicales et à la Faculté de Santé Publique } \\
\hline Niveau (CECRL) et durée & \multicolumn{3}{|c|}{ B1 - 2h30 } \\
\hline Tâche finale & \multicolumn{3}{|c|}{$\begin{array}{l}\text { Ecrire une lettre officielle au Ministre de la Santé pour optimiser la qualité des soins et promouvoir les } \\
\text { hôpitaux de campagne en temps de COVID-19. }\end{array}$} \\
\hline Objectifs pragmatiques & \multicolumn{3}{|c|}{$\begin{array}{l}\text { Etre capable de/d': } \\
\text { - } \quad \text { interagir sur des thématiques en lien avec les hôpitaux de campagne. } \\
\text { - } \quad \text { exposer en connaissance de cause le rôle que doivent jouer les hôpitaux de campagne. } \\
\text { - } \quad \text { exprimer sa méfiance/confiance au sujet des conditions sanitaires et sécuritaires dans les } \\
\text { hôpitaux de campagne. }\end{array}$} \\
\hline Objectifs linguistiques & \multicolumn{3}{|c|}{$\begin{array}{ll} & \text { Etre capable d'utiliser : } \\
\text { - } & \text { le lexique médical en lien avec la COVID-19. } \\
\text { - } & \text { les expressions de la cause, de la concession, de la comparaison. } \\
\text { - } & \text { les adjectifs et les pronoms indéfinis. }\end{array}$} \\
\hline Objectifs socio/inter/culturels & \multicolumn{3}{|c|}{$\begin{array}{l}\text { - } \quad \text { connaître les règles de rédaction de la lettre formelle. } \\
\text { - } \quad \text { adapter son discours à son interlocuteur (utiliser un langage soutenu, le vouvoiement, etc.) } \\
\text { - } \quad \text { comprendre la différence entre les conditions/services des hôpitaux de campagne à différentes } \\
\text { époques de l'histoire. } \\
\text { - } \\
\text { comparer les hôpitaux de campagne dans différents pays et à partir de supports divers ( } \\
\text { reportages, œuvres d'art) }\end{array}$} \\
\hline \multirow{2}{*}{ Habiletés langagières } & \multirow{2}{*}{ Situation } & \multicolumn{2}{|c|}{ Document(s) } \\
\hline & & Support & Outils numériques \\
\hline Comprendre & $\begin{array}{l}\text { Document sonore/audiovisuel sur } \\
\text { l'actualité sanitaire en lien avec la } \\
\text { COVID-19 }\end{array}$ & $\begin{array}{l}\text { Vidéo : Un premier hôpital } \\
\text { de campagne à Londres } \\
\text { https://www.youtube.com/w } \\
\text { atch?v=MXEB6XiZdpk }\end{array}$ & Edpuzzle \\
\hline
\end{tabular}




\begin{tabular}{|c|c|c|c|c|}
\hline & Lire & $\begin{array}{l}\text { Article de presse relatif à l'installation } \\
\text { des hôpitaux de campagne au Liban. }\end{array}$ & $\begin{array}{l}\text { Coronavirus : un hôpital de } \\
\text { campagne installable en } 20 \\
\text { minutes arrive à Bayonne } \\
\text { https://www.europe1.fr/socie } \\
\frac{\text { te/coronavirus-un-hopital-de- }}{\text { campagne-pret-en-20- }} \\
\text { minutes-a-bayonne-4004480 }\end{array}$ & $\begin{array}{l}\text { TEDEd (rubrique Think) } \\
\text { Google Forms }\end{array}$ \\
\hline \multirow[t]{2}{*}{ Parler } & $\begin{array}{l}\text { Prendre part à une } \\
\text { conversation }\end{array}$ & $\begin{array}{l}\text { Echanger avec des étudiants au sujet } \\
\text { des hôpitaux de campagne pour } \\
\text { optimiser leur fonctionnement }\end{array}$ & $\begin{array}{l}\text { Toile : Un hôpital italien } \\
\text { surchargé pendant } \\
\text { l'épidémie de grippe } \\
\text { espagnole de 1918-1919 } \\
\text { (anonyme) }\end{array}$ & Zoom / Microsoft Teams \\
\hline & $\begin{array}{l}\text { S'exprimer } \\
\text { oralement en continu }\end{array}$ & $\begin{array}{l}\text { Raconter dans la page Facebook de } \\
\text { l'UL le défi auquel fait face le corps } \\
\text { médical durant la COVID-19 }\end{array}$ & $\begin{array}{l}\text { Toile : Tribute to Heros } \\
\text { (Leila Dagher) }\end{array}$ & Active Presenter \\
\hline Ecrire & \multicolumn{2}{|c|}{$\begin{array}{c}\text { Ecrire une lettre officielle au Ministre de la Santé pour } \\
\text { optimiser la qualité des soins offerte en temps de COVID-19 }\end{array}$} & $\mathrm{X}$ & $\mathrm{B} \log$ \\
\hline
\end{tabular}

En définitive, cette offre de cours à distance est à expérimenter lors de prochaines formations. Elle est constituée d'activités toutes interdépendantes, agencées selon une progression fonctionnelle qui consolident les quatre habiletés langagières. 


\section{Conclusion}

Dans cet article, nous avons souhaité nous interroger sur le rôle et la place des enseignants de français dans l'enseignement en ligne post-COVID. Nos résultats ont montré que l'introduction de nouvelles techniques d'enseignement et d'un nouveau curriculum prenant en considération le contexte de crises sont plébiscités par une majorité d'enseignants. Dans cette perspective, notre recherche a su montrer que le changement de pratique professionnelle est un processus qui pourrait se faire par le biais des arts plastiques dans la mesure où ils facilitent, d'une part, l'accès à la langue cible et, d'autre part, sont un véritable catalyseur de motivation et d'interactions entre apprenants et enseignants. En effet, toute la production artistique générée après la COVID-19 propose à l'enseignant un panel de documents authentiques pour créer un matériau pédagogique sur mesure. De plus, le choix des logiciels numériques est large pour concevoir des activités riches, variées et adaptées aux différents contextes académiques des apprenants.

À notre avis, le projet d'intégration de nouveaux curricula dans le système universitaire public mérite l'établissement d'une stratégie bien définie basée sur un référentiel de compétences.

Ainsi, cette étude a permis de saisir les tendances actuelles en termes d'innovation pédagogique. Le passage de la réflexion à l'action est l'occasion d'innover en matière de formation. La mise en place des propositions de formation susmentionnées est envisageable en adoptant une attitude holistique qui est la voie d'entrée vers une démarche systémique prenant en considération tous les aspects de la problématique de l'enseignement/apprentissage du français en tant qu'action située.

\section{References:}

1. Carey, K. (2016). The End of College. Creating the Future of Learning and the University of Everywhere. New York : Riverhead Books.

2. Cuban, L. (1999). How scholars trumped teachers. Change without reform in university curriculum, teaching, and research, 1890-1990. New York : Teachers College Press, Columbia University.

3. Klein, C. (2013). Les usages du numérique pour l'enseignement du FLE/FLS/FLSCO. L’école numérique, (16, juin 2013), 8-11.

4. Le Robert. (1994). Dictionnaire historique de la langue française. Paris : Ed. du Club France Loisirs.

5. Longuet, F. et Springer, C. (2012). Développer et évaluer les compétences professionnelles des enseignants de langues à l'université : une mission impossible ? Dans M. Causa (dir.), Formation initiale et profils d'enseignants de langues. Enjeux et questionnements (p. 247278). Bruxelles : De Boeck Supérieur. 
6. Tremblay, N-A. (2003). L'autoformation : Pour apprendre autrement. Nouvelle édition [en ligne]. Montréal : Presses de l'Université de Montréal, (généré le 18 janvier 2021). Lien : http://books.openedition.org/pum/10719

7. Schön, D. A. (1983). The Reflective Practitioner. How Professionnals Think in Action. New York, NY : Basic Books.

8. Witzigmann, S. (2014). L'art dans tous ses états, synergies entre le français langue étrangère et les arts plastiques, $\mathrm{N}^{\circ} 6,2014.5$ Editions du CRINI (C e-crini. ISSN 1760-475.

9. Erdekian, A. (26 mai 2020). The Coronavirus Street Art Helping Lift Spirits Around the World. Lien : https://www.cntraveler.com/gallery/coronavirus-street-art

10. Ricci, B. (2020). Coronavirus Street Art: How The Pandemic Is Changing Our Cities. https://magazine.artland.com/coronavirus-street-art-how-thepandemic-is-changing-our-cities/

11. Bureau du traducteur. (11 juin 2020). Lexique sur la pandémie de COVID-19.

Lien : https://www.btb.termiumplus.gc.ca/publications/covid19-fra.html

12. Cahiers de l'IPM, (2005). Les 10 compétences de l'enseignant universitaire, $\quad \mathrm{n}^{0} 5, \quad$ p.11. Lien : https://aurelieferon.files.wordpress.com/2017/09/ipm_complementrdc-aipu-1999.pdf

13. Raby, C. et al. (2004). Le développement de la compétence professionnelle des enseignants du préscolaire et du primaire à intégrer les TIC en classe : impact d'une recherche-action. Lien : https://formation-profession.org/files/numeros/5/v21_n02_2.pdf

14. Eddé, C. (4 avril 2020). L'enseignement en ligne vu par les étudiants. OLJ. Lien : https://www.lorientlejour.com/article/1213216/lenseignement-enligne-vu-par-les-etudiants.html

15. Frau-Meigs, D. (4 mai 2020). Pédagogie à distance : les enseignements du e-confinement. Lien : https://theconversation.com/pedagogie-adistance-les-enseignements-du-e-confinement-137327

16. El Hage, A-M. (16 mars 2020). L'enseignement en ligne pour tenter de sauver l'année scolaire au Liban. Lien : https://www.lorientlejour.com/article/1210583/lenseignement-enligne-pour-tenter-de-sauver-lannee-scolaire-au-liban.html

17. Equipe Erasmus + des Experts pour la réforme de l'enseignement supérieur (HERE) au Liban. (2020). Guide pour l'apprentissage et l'enseignement en ligne Le contexte de la pandémie Coronavirus. Lien : http://erasmusplus- 
lebanon.org/sites/default/files/documents/Guide_Online_T\%26L_at_ Coronavirus_Context_FR.pdf

18. Jarraud, F. (27 novembre 2020). Philippe Meirieu : Enseigner : un métier de résistant. L'Express. Café pédagogique. Lien : http://www.cafepedagogique.net/lexpresso/Pages/2020/11/27112020 Article637420575527675818.aspx

19. Gac, R. (2007). Braun-Vega, maître de l'interpicturalité. Lien : http://sens-public.org/articles/376/

20. Haddad, G. (30 octobre 2020). Epidemics and the Arts. Lien : https://www.aub.edu.lb/Events/Pages/Details.aspx?ItemId=3086

21. Institut français de l'éducation. (Consulté le 4 décembre 2020). Mettre en place / suivre un cours à distance. Lien: http://neosup.enslyon.fr/app.php/situation/9/theme/39

22. Naciri, K. (11 août 2020). Confinement : les universités marocaines à l'épreuve de l'Enseignement à distance. Lien : https://www.youtube.com/watch?v=zSfUJmTrFJo

23. Messai-Farkh, S. et Kayal, J. (2019). L'intégration des technologies numériques et méthodes pédagogiques innovantes dans le système universitaire : le cas de l'Université Libanaise. Dans S. NicolaïdèsSalloum, I. Slim-Hoteit (Éds.). Le numérique dans les modèles éducatifs : réalités, défis et perspectives. Lien: https://www.amazon.fr/num\%C3\%A9rique-dans-mod\%C3\%A8les\%C3\%A9ducatifs-perspectives-ebook/dp/B08LZK9WFQ

24. Ministère de l'Éducation et de l'Enseignement supérieur québécois. (2019). Cadre de référence de la compétence numérique. Lien: http://www.education.gouv.qc.ca/fileadmin/site_web/documents/mini stere/Cadre-reference-competence-num.pdf

25. Brassard, N. (2012). Profil de compétences de l'enseignant de niveau universitaire, ENAP. Lien: http://www.uqac.ca/cpu/wpcontent/uploads/2014/10/Profil_comp\%C3\%A9tences_brassard.pdf

26. Nations Unies. (Août 2020). Note de synthèse : L'éducation en temps de COVID-19 et après. https://www.un.org/sites/un2.un.org/files/policy_brief__education_during_covid-19_and_beyond_french.pdf

27. Perrenoud, P. (1997). Construire des compétences dès l'école. Paris : ESF éditeur. Lien : https://www.erudit.org/fr/revues/rse/1998-v24-n2rse1839/502035ar.pdf

28. République Algérienne Démocratique et Populaire Ministère de l'Enseignement Supérieur et de la Recherche Scientifique. (2016). Référentiel de compétences des enseignants-chercheurs nouvellement recrutés.

Lien :

https://www.univ-chlef.dz/uc/wp- 
content/uploads/pdf/Referentiel-de-competences-des-enseignantschercheurs.pdf

29. Renassia, S. (31 mars 2020). Haddock pète les plombs en confinement : les albums de Tintin détournés avec humour. Lien : https://positivr.fr/des-internautes-detournent-de-celebres-albums-detintin-version-confinement-9-images/

30. Syndicat national de l'enseignement supérieur, Fédération syndicale unitaire. (2018). Référentiel métier des enseignants chercheurs, Document de travail du MESRI - Version du 11 avril 2018, soumise à concertation. Lien : https://www.snesup.fr/article/referentiel-metierdes-enseignants-chercheurs

31. Karensti, T. et al. (2004). Les futurs enseignants du Québec sont-ils bien préparés à intégrer les TIC ? Lien : https://www.researchgate.net/publication/267035198_Les_futurs_ens eignants_du_Quebec_sont-ils_bien_prepares_a_integrer_les_TIC

32. Libaert, T. (2018). Communication de crise, Pearson. Lien : https://www.pearson.fr/resources/titles/27440100209020/extras/F017 0_Chap1.pdf

33. Mitman, T. (18 mai 2020). Coronavirus murals: inside the world of pandemic - $\quad$ inspired street art. Lien : https://theconversation.com/coronavirus-murals-inside-the-world-ofpandemic-inspired-street-art-138487

34. Unicef. (Avril 2020). Éducation COVID-19 : Cadre pour la planification de contingences, réduction des risques, préparation et réponse. New York. Lien : https://www.unicef.org/lac/media/12351/file

35. Unicef. (Mars 2020). Messages clés et actions pour la prévention et le contrôle de la COVID-19 dans les écoles. Lien : https://www.unicef.org/media/65846/file/Key\%20Messages\%20and $\% 20$ Actions $\% 20$ for $\% 20$ COVID-

19\%20Prevention\%20and\%20Control\%20in\%20Schools_French.pdf

36. Université Libanaise. (2020). Enseigner à distance. Lien : https://www.ul.edu.lb/ul/mteams/default.aspx?lang=3

37. Université de Saint Joseph de Beyrouth. (2020). Aspects pédagogiques de l'enseignement en ligne. Lien : https://www.usj.edu.lb/news.php?id=9560\&fbclid=IwAR31EMqkWYNX-RU-idlf-g-WyfM74Wen1pWo1c_2AE_eEcUu_57D_GsKg

38. Zeitoun, S. et Ghosn, R. (2018). Compétences Des Enseignants Universitaires : Etude De Cas Du Centre Des Sciences Du Langage Et De La Communication (CSLC) À L'Université Libanaise. Tripoli : Université Al Jinane. Lien : 
39. https://www.researchgate.net/publication/333552867_Competences Des_Enseignants_Universitaires_Etude_De_Cas_Du_Centre_Des_Sc iences_Du_Langage_Et_De_La_Communication_CSLC_A_L'Unive rsite_Libanaise 